\title{
Gestión de información personal con software para mapas conceptuales
}

\author{
Por Jesús Tramullas, Ana-I. Sánchez-Casabón y Piedad Garrido-Picazo
}

\begin{abstract}
Resumen: La gestión de información personal (Personal information management, PIM) plantea problemas debido a que el software que se utiliza no parece responder adecuadamente a los comportamientos y actividades de los usuarios. Se revisa el concepto y problemas de la gestión de información personal, y las herramientas de software que se utilizan en la misma. Se evalúa cualitativamente una aproximación basada en el uso de aplicaciones de software para mapas conceptuales como respuesta a los problemas planteados.

Palabras clave: Gestión de información personal, Comportamiento de usuarios, $\mathrm{He}$ rramientas de software, Mapas conceptuales.
\end{abstract}

Title: Personal information management using concept mapping software

Abstract: Personal information management (PIM) raises some problems because the software

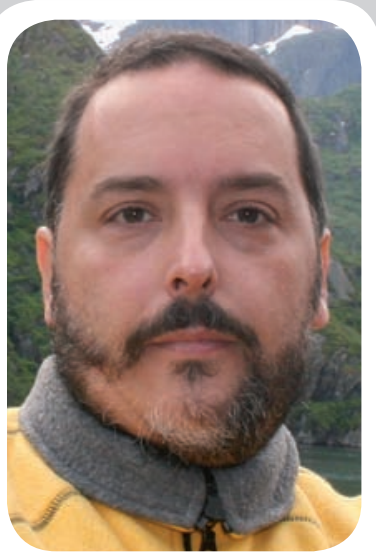

Jesús Tramullas es profesor titular en el Departamento de ciencias de la documentación de la Universidad de Zaragoza y miembro del grupo de investigación sobre Gestión de recursos de información en las organizaciones (GRIO). Es investigador principal de diferentes proyectos, como "Web semántica y bibliotecas digitales: desarrollo de servicios de información basados en rdf y topic maps" (2006-2007). Sus líneas de investigación se centran en gestión personal de información, bibliotecas digitales y servicios de información digital, y herramientas de software libre para la gestión de información.

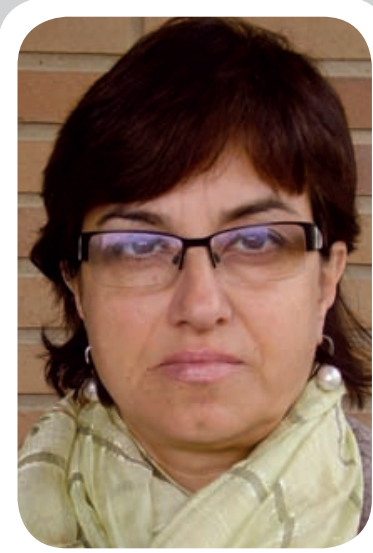

Ana-I. Sánchez-Casabón es doctora en historia medieval. Profesora titular en el Departamento de ciencias de la documentación en la Universidad de Zaragoza. Imparte docencia en el Grado de información y documentación y en el Master en Gestión de unidades y servicios de información y documentación. Miembro del grupo de investigación sobre Gestión de recursos de información en las organizaciones (GRIO). Sus líneas de investigación están relacionadas con la alfabetización información y el marketing y difusión de servicios de información. Ha participado en varios proyectos de investigación.

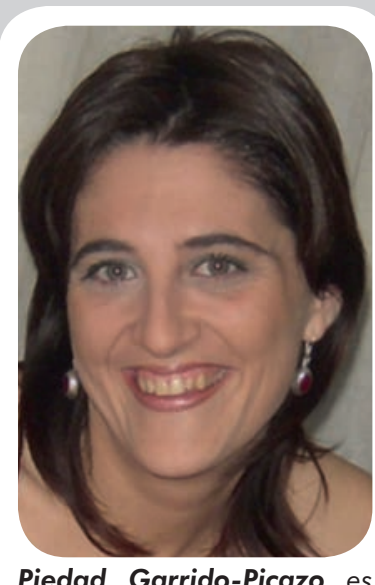

Piedad Garrido-Picazo es doctora en documentación por la Universidad Carlos III de Madrid. Profesora ayudante doctor en el Departamento de informática e ingeniería de sistemas de la Universidad de Zaragoza. Miembro del grupo de investigación sobre Gestión de recursos de información en las organizaciones (GRIO). Ha publicado diferentes trabajos en revistas nacionales e internacionales. Sus líneas de investigación se relacionan con bases de datos $x \mathrm{ml}$, software libre para gestión de información, bibliotecas digitales, rdf y topic maps (XTM) en el contexto de la web semántica, y calidad del software.

tools that are used don't adequately respond to user behaviour and activities. The concept and personal information management problems are reviewed, along with the software tools used for this task. A qualitative assessment explores whether an approach based on software tools for conceptual maps can address the problems discussed.

Keywords: Personal information management, User behaviour, Software tools, Concept maps.

Tramullas, Jesús; Sánchez-Casabón, Ana-I.; Garrido-Picazo, Piedad. "Gestión de información personal con software para mapas conceptuales". El profesional de la información, 2009, noviembre-diciembre, v. 18, n. 6, pp. 601-612.

DOI: 10.3145/epi.2009.nov.03

\section{La gestión de información personal (PIM)}

Los usuarios de sistemas y servicios de información digital han adoptado durante la última década actitudes activas en la gestión de la información que utilizan para alcanzar sus objetivos y satisfacer sus necesidades. La proliferación de recursos de información digital que están sustituyendo a los tradicionales en contenidos y formatos, la creciente disponibilidad de aplicaciones, así como los nuevos servicios de la web 2.0, han facilitado las tareas que se pueden llevar a cabo sin un intermediario o profesional especializado. Por ello la desintermediación en el acceso y la gestión de información se ve como consecuencia de la progresión de las habilidades adquiridas por los usuarios y de la disponibilidad de herramientas de software, gratuitas y/o libres, que las potencian. En este contexto cada vez 
adquiere mayor importancia la gestión de información personal (Personal information management, PIM).

\section{"La desintermediación en el acceso y la gestión de información es consecuencia de la progresión de las habilidades de los usuarios y de la disponibilidad de software"}

La investigación sobre la gestión de información personal (en adelante, PIM) es objeto de creciente interés en la primera década de este siglo (Jones; Teevan, 2007; Jones, 2007a). ACM le dedicó en 2006 uno de los números de Communications of the ACM, (v. 49, n. 1) y en 2008 un número de ACM Transactions on information systems (v. 26, n. 4). Una de las primeras características a destacar de la PIM es que, al igual que en otros sectores científicos que nos ocupan, se trata de un término genérico, sobre el cual Boardman señala: "The term Personal Information Management, often abbreviated to PIM, is used as an umbrella term to describe the everyday process performed by individuals as they collect, store, organize and access their collections of digital objects". (Boardman, 2004: 13). En este término intervienen enfoques de interacción persona-ordenador e interfaces de usuario, gestión de información, recuperación de información, elaboración de software y gestión de proyectos. En su análisis de diferentes definiciones, el mismo autor señala la existencia de una perspectiva "tradicional", para la cual el principio básico es que la información se almacena para ser posteriormente recuperada. En una segunda aproximación, la PIM muestra como factor fundamental la actuación de los objetos de información como recordatorios y disparadores de tareas que deben llevarse a cabo. Tras este análisis, Boardman propone como definición de información personal: "[...] information owned by an individual, and under their direct control"; y como definición de PIM: "the management of personal information as performed by the owning individual" (2004: 15, 16), ambas muy amplias, como puede apreciarse.

Frente a otras definiciones, el modelo de Boardman conceptualiza la PIM como una actividad individual del usuario, en el marco de la cual lleva a cabo tareas de adquisición, organización, mantenimiento y recuperación de elementos de información dentro de colecciones, todo ello con la ayuda de software. Desde una perspectiva operacional, Jones (2007b) adopta la formulación clásica: "Personal Information Management (PIM) refers to both the practice and the study of the activities people perform in order to acquire, organize, maintain and retrieve information for everyday use", y propone un marco de trabajo con tres tareas esenciales, correspondientes a recopilación, búsqueda/ re-búsqueda y mantenimiento (que denomina $M$-Level activities, e incluye el mapping u organización de la información). Barreau et al. (2008) hablan de finding, keeping y refinding como las tres actividades principales, detallando los problemas que plantea su realización en entornos abiertos o cerrados, así como la gran cantidad de factores que afectan especialmente al refinding, la actividad más problemática para su estudio y análisis.

Debe contrastarse esta aproximación con el enfoque clásico que limita la PIM a la búsqueda y recogida de información en internet, y a las actividades para volver a encontrarla posteriormente (Bruce; Jones; Dumais, 2004). Otra vía de investigación se ha limitado a analizar la forma en que los usuarios organizan sus ficheros en los escritorios o sistemas de archivos de los ordenadores (Khoo et al., 2007). Independientemente de las posturas que adopten los diferentes investigadores, en el momento actual se acepta que la PIM es eminentemente operacional, individual, mediada tecnológicamente, y su material de trabajo es cualquier tipo de información digital.

Diferentes autores establecen tres niveles a considerar: ítems, elementos u objetos de información; colecciones; y espacios de información. Para el análisis que nos ocupa, un ítem, elemento u objeto, sería una unidad informativa con sentido completo, independiente de otras (por ejemplo, un correo electrónico, una imagen, un documento ofimático, un marcador, una nota, o una lista de tareas). Los objetos de información poseen una forma asociada, en este caso el formato digital en el cual se encapsula la información de contenido.

Una colección de información personal es para Bruce (2005) una expresión que hace referencia a la organización personal y a la perspectiva de la información de un individuo, e incluye objetos o elementos de información, estructuras para representar y organizar la información, y puntos de acceso, como punteros o enlaces. Todo ello considerado siempre desde el entorno cognitivo del usuario: "It is a collection of information sources and channels that we as individuals have acquired, cultivated, and organized over time and in response to a range of stimuli. The personal information collection is an organic and dynamic personal construct that we take with us into, and out of, the various information events that frame our daily working and personal lives". Se trata por tanto de una construcción intelectual, personal, influenciada por las experiencias y contextos en los que los usuarios llevan a cabo su actividad. 
McKnight (2000) utilizó la expresión "espacio de información" para referirse a las colecciones de información personal. Jones (2007b) habla de un "espacio de información personal", en el cual integra todos los objetos de información y las colecciones en las que participan, pero también las aplicaciones de software que sirven para su adquisición, organización, mantenimiento y recuperación.

\section{Usuarios y PIM}

El marco de trabajo general en el estudio de la PIM que llevan a cabo los usuarios, pretende analizar las tareas que éstos realizan, sus comportamientos ante la información digital, y la manera en que utilizan el software. Boardman y Sasse (2004) ofrecen una revisión de estudios de comportamientos de usuarios. Para el análisis de las actividades de los usuarios se han usado aproximaciones desde el análisis del trabajo cognitivo, teoría de la actividad, de arquitecturas cognitivas, y del denominado sense-making. Por ejemplo, Jones (2007b) propone un modelo con tres actividades básicas:

- De la necesidad a la información: la búsqueda de información nueva, debida a actividades que requieren tomar decisiones, ejecutar tareas, o aumentar conocimientos.

- De la información a la necesidad: en el marco de una actividad, el usuario encuentra en su espacio de información personal, información reunida con anterioridad pero que ahora es redescubierta, o toma valor en cuanto puede ser utilizada en otros contextos o en situaciones futuras.

- Las correspondencias entre necesidad e información: equivalen a lo que en un párrafo anterior se ha identificado como $M$-level activities. Se trataría de las funciones de clasificación y organización de los objetos y colecciones de información en el espacio de información personal.

\section{"Uno de los problemas de los usuarios son sus fallos de memoria cuando deben recordar de qué manera y en qué lugar guardaron información que necesitan"}

El citado trabajo de Jones revisa numerosa bibliografía sobre la cuestión, y de él puede deducirse que la actividad más compleja y que genera mayores problemas entre usuarios es la segunda de ellas, dado el creciente volumen de información disponible, la naturaleza fragmentaria de la misma, y la concepción borrosa de los procesos de reutilización y replicado de los objetos de información por parte de los usuarios. Algunos trabajos incluso indican la posibilidad de "no hacer nada" como una opción común que toman en consideración los usuarios. Boardman y Sasse (2004) tras controlar y analizar el comportamiento de un grupo de usuarios y sus estrategias longitudinales en diferentes aplicaciones, en especial sobre contenidos de ficheros, correo electrónico y marcadores, concluyeron que los usuarios tendían a trabajar más la organización de ficheros que de correo electrónico o marcadores, por su origen, forma de adquisición y posibilidades de reutilización de la información. De la misma forma algunas tareas de gestión no requerían aplicaciones complicadas con alto nivel de integración, al tiempo que los usuarios tenían diferentes necesidades de organización en diferentes herramientas. En cualquier caso, los autores señalaban que los cambios en comportamientos se producían a medio y largo plazo. Hoy en día, con la explosión de las aplicaciones 2.0, cabría plantearse si los cambios en las pautas de gestión se mueven ya en el corto plazo.

Elsweiler, Ruthven y Jones (2007) han señalado que uno de los problemas a los que se enfrentan los usuarios son los fallos de memoria cuando deben recordar de qué manera y en qué lugar guardaron información que necesitan para una tarea en un momento dado. Se producen errores de memoria retrospectiva, de memoria prospectiva y errores de acción. Los usuarios usan diferentes tipos de memoria, dependiendo del momento e interés de la recogida y de la necesidad de información, y para mejorar los sistemas de PIM es necesario estudiar qué olvidan y por qué.

Jones (2004) ha estudiado el impacto que la decisión de los usuarios tiene en los costes de mantenimiento y recuperación de información, y las diferentes pautas que se pueden seguir en virtud de los diferentes contextos y usos de software. Como ejemplo de ello, baste citar el reciente trabajo de Bergman et al. (2008), que demuestra que los usuarios llevan a cabo búsquedas locales en sus colecciones y espacios de información personal principalmente mediante exploración y navegación, con preferencia a usar motores de búsqueda local, independientemente de su capacidad para mostrar resultados relevantes. Gwizdka y Chignell (2007) han defendido que, cuando se llevan a cabo estudios de usuarios, en primer lugar deben analizarse el medio ambiente y el contexto de las tareas que realizan, para pasar posteriormente a aspectos grupales y por último, individuales. Estas conclusiones sirven para ilustrar las diferentes pautas de conducta o comportamiento informacional de los usuarios dependiendo de su contexto. Como planteamientos generales puede aceptarse que:

- La actividad de cada usuario es única, contextual, y personal. 
- Los fallos de memoria afectan a la gestión de información.

- La actividad se lleva a cabo en entornos heterogéneos, con varias herramientas, formatos...

- El ciclo de vida de la información personal es impredecible, no sigue pautas fijas, y es dinámico y cambiante.

\section{Herramientas de software para PIM}

Boardman (2004: 19) las define como "[...] a software tool that allows a user to manage a collection of personal information items. The PIM-tool interface defines how a user views and interacts with the collection". Este autor distingue entre las orientadas específicamente para gestionar algún tipo de información personal, y aquellas en las que esta gestión es secundaria. Como herramientas típicas señala los sistemas de ficheros, la mensajería electrónica, los marcadores, las agendas y calendarios, los gestores de contactos, las listas de tareas, etc., e incluye las aplicaciones para colecciones de música, de fotografías y de referencias. Esta enumeración sirve para destacar algunos aspectos del estado actual de las herramientas:

- No hay una definición precisa de las particularidades que diferencian el software para PIM de otros tipos de software que los usuarios utilizan, total o parcialmente, para el mismo fin.

- No hay una integración pautada de aplicaciones mediante protocolos de intercambio, ni de intercambio de información mediante estándares de formato o etiquetado

- Estas carencias obligan a los usuarios a llevar a cabo la PIM mediante la utilización de diferentes herramientas de software.

Si se toman como punto de partida las definiciones y peculiaridades de la PIM, el software debe ofrecer soporte para las tareas de:

- Adquisición de objetos de información.

- Organización de información tanto en lo que se refiere a las colecciones, como a los elementos u objetos que conformen las colecciones.

- Gestión de colecciones y de elementos constituyentes.

- Recuperación de información.

Una revisión de los listados de software disponible bajo la denominación Personal Information Manager permite apreciar que no existe un consenso, ya que hay desde gestores de correo con extensiones de agenda, a sistemas de notas, gestores de marcadores, aplicaciones de directorio, calendarios, buscadores locales de contenido de documentos, o combinaciones de algunos tipos diferentes. También hay usuarios que usan sistemas de gestión de contenidos, o wikis personales. A ello debe añadirse que, debido al auge y expansión de servicios de la web 2.0, los usuarios han pasado a delegar aplicaciones y contenidos típicos del tratamiento de la información personal en servicios web. Todo esto muestra un panorama cada vez más heterogéneo, con las ventajas o inconvenientes que conlleva. Esta situación dificulta establecer un tipo de herramientas para PIM, dada la variedad de estrategias que adoptan los usuarios.

Como consecuencia del dinamismo señalado en los objetos de información digital y las colecciones en las que se organizan, así como la variabilidad en los objetivos y necesidades del usuario, se entiende que las aplicaciones deben incorporar dentro de sus funcionalidades la posibilidad de reflejar esa variabilidad en la actualización de la información, y en la integración de objetos de información en diferentes colecciones de manera simultánea.

Boardman introduce la noción de Personal Information Environment (entorno de información personal), que define como: "[...] the aggregate of all collections of personal information", pero lo importante es que considera dentro del mismo tanto las colecciones situadas en la máquina del usuario, como a las almacenadas en máquinas remotas, y las situadas dentro de dispositivos móviles (2004: 26). Una de las cuestiones que se desprende de los enfoques de entorno o escritorio es que las aplicaciones deberían ofrecer algún nivel de integración. Ésta puede llevarse a cabo atendiendo a varias aproximaciones:

- Integración por interfaz de búsqueda: mediante el uso de una única interfaz de búsqueda para todos los tipos de contenidos.

- Integración por agrupación: mediante una interfaz que ofrezca al usuario la posibilidad de agrupar o filtrar los objetos según diferentes criterios.

- Integración por vista de información: mediante una visión gráfica que integre todos los elementos u objetos de una colección.

Como ejemplo clásico de herramienta para PIM puede citarse el prototipo Stuff I've Seen (Cutrell; Dumais; Teevan, 2006). Se trata de una aplicación de escritorio que busca en los contenidos digitales del ordenador de sobremesa del usuario, y presenta como respuesta una interfaz que provee acceso integrado a los resultados de la búsqueda, independientemente del tipo de documento que se trate (correo electrónico, documentos, marcadores, etc.). Como novedad, incorpora un control de visualización por línea de tiempo, dada 


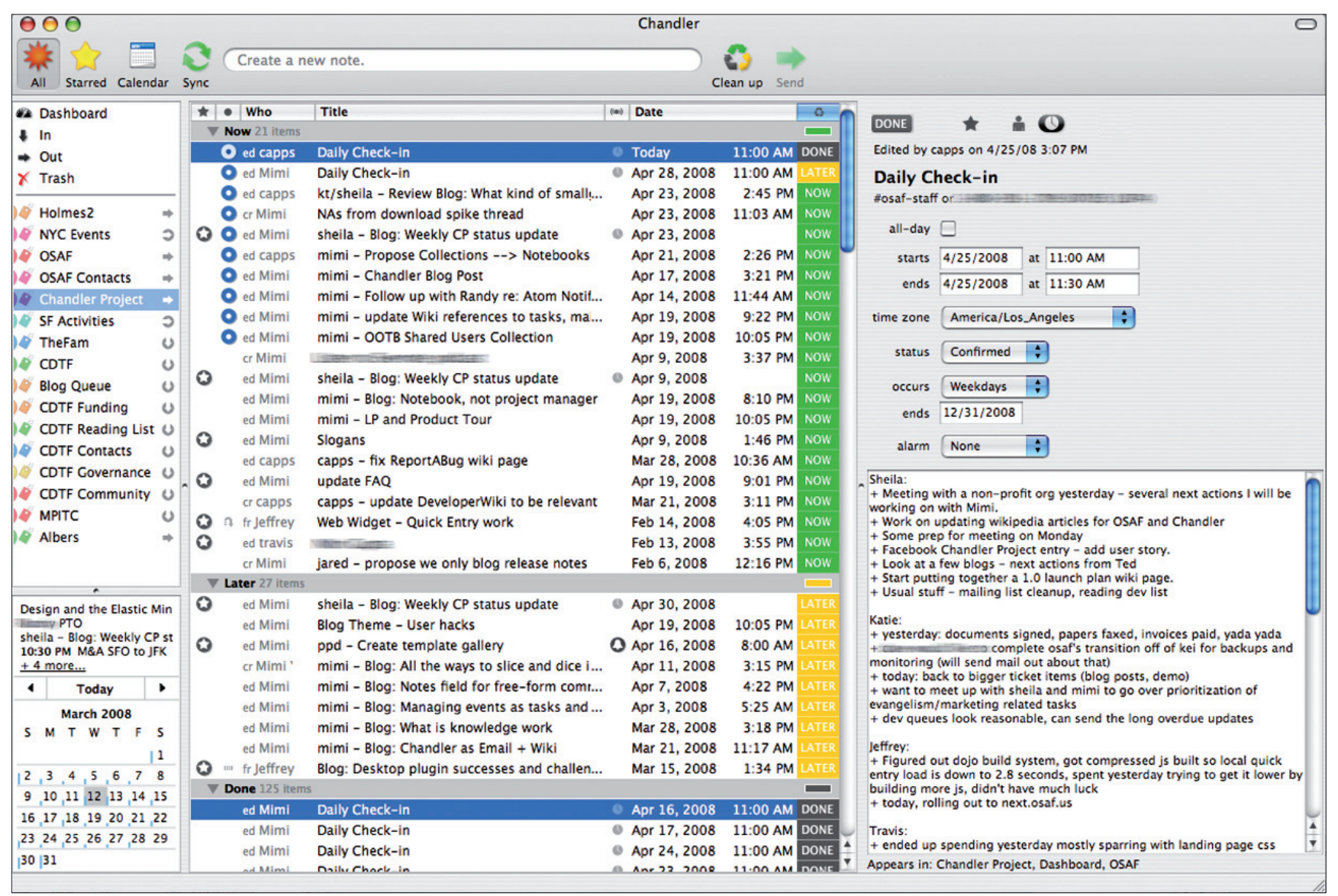

Figura 1. Chandler, un gestor de información personal http://chandlerproject.org

la tendencia de los usuarios a recordar información y datos por etapas temporales asociadas. Además, en las últimas versiones incorpora extensión automática y recomendación de búsqueda gracias a la detección de metadatos y términos significativos en la respuesta obtenida. Otro enfoque es el empleado por aplicaciones que usan estandarización semántica para etiquetar y relacionar los contenidos informativos (Karger; Jones, 2006). Haystack fue un prototipo de investigación en el que se optó por el uso de xml y rdf como elementos básicos de descripción de objetos informativos y de sus relaciones. Por encima de la capa semántica, se ofrecía una interfaz tradicional de acceso y organización de la información, basada en la asociación y navegación entre objetos de información.

Bergman, Beyth-Marom y Nachmias (2003) han señalado que para el diseño de sistemas de PIM adecuados, deberían tenerse en consideración tres principios básicos:

- Principio de clasificación subjetiva: los objetos de información relacionados con el mismo tópico (colección) deberían clasificarse juntos independientemente de su formato digital.

- Principio de importancia subjetiva: la importancia subjetiva de la información que se trate debería determinar su nivel de relevancia visual y accesibilidad.
- Principio de contexto subjetivo: la información debería ser recuperada y visualizada por el usuario en el mismo contexto en el que la usó previamente.

En un trabajo posterior (Bergman; Beyth-Marom; Nachmias, 2008) han concluido que los sistemas actuales no dan un soporte adecuado al factor de subjetividad de los usuarios cuando organizan la información personal, ya que las herramientas disponibles se ajustan más al uso de atributos objetivos, como proyecto, importancia y contexto.

Es necesario tener en cuenta que toda la información digital que se usa en procesos personales de decisión o trabajo no puede ser adecuadamente gestionada con las aplicaciones disponibles. Bernstein; Van-Kleek; Karger; Schraefel (2008) han identificado objetos de información heterogéneos a los que han denominado information scraps (recordatorios, post-it, notas, listas de todo, mensajes cortos, etc.) y que suelen contener información y datos no usuales, en el marco de un ciclo de vida temporal y específico, pero que desempeñan un papel fundamental en la realización de las tareas y actividades de los usuarios. La elaboración de pequeños complementos, o widgets que pueden integrarse en los escritorios de los actuales sistemas operativos, especialmente en entornos GNU/Linux y Mac OS X, por ejemplo mediante dockbars, hace posible que se 
pueda delinear una primera aproximación a entornos de gestión de información personal en los cuales pequeñas aplicaciones especializadas se pueda combinar y agregar de manera dinámica para crear un espacio de información personal ajustado a las necesidades del usuario.

Las tecnologías semánticas han sido propuestas como una solución para integrar de manera consistente la PIM. Sauermann (2005) ha propuesto un prototipo de Semantic Desktop, llamado Gnowsis, que es un entorno de escritorio potenciado mediante técnicas de web semántica, y en el cual las aplicaciones y su contenido se relacionan y enlazan mediante etiquetado en rdf y ontologías en owl.

\section{"Los mapas de conceptos son una técnica que pretende representar conocimiento mediante grafos"}

\section{Mapas conceptuales como técnica de organización de conocimiento e información}

Los mapas de conceptos son una técnica que pretende representar conocimiento mediante grafos. Fueron elaborados en la década de 1960 por Joseph Novak en la Cornell University (Novak; Cañas, 2008). En su formulación clásica, los nodos representan conceptos y los arcos o enlaces entre ellos reflejan las relaciones entre los conceptos a los que unen. La teoría establece que los conceptos y los enlaces pueden ser de diferentes tipos, atendiendo a la información que reflejan, a las asociaciones que establecen y a otros criterios como espacio, causa o tiempo. Los mapas de conceptos han sido utilizados para describir los sistemas hipertextuales como espacios de organización y de navegación entre documentos (Colomb, 2002). Una técnica relacionada, y ampliamente difundida en diferentes entornos, es la conocida como Mind Mapping, que ha sido además registrada como marca y como producto por su creador, T. Buzan (2000). Esta técnica pone el énfasis en la representación visual rápida de pensamientos $\mathrm{y}$ anotaciones en procesos cognitivos, alrededor de un concepto central.

Si bien en un primer momento la aplicación de los mapas de conceptos se centró en entornos educativos, su aplicación se ha extendido a espacios no educativos y están generando en la actualidad una gran actividad científica interdisciplinar en su entorno. Se están convirtiendo en instrumentos ideales para la navegación conceptual guiada ya que facilitan el acceso a nume- rosos recursos informativos previamente organizados, o sobre los cuales se lleva a cabo una organización y acceso diferentes a lo previamente existente. Los mapas conceptuales permiten generar estructuras de representación del conocimiento más complejas, y organizar el mismo alrededor de varios conceptos o nodos nucleares, adoptando formas jerárquicas o radiales más profundas. Los componentes nucleares de un mapa conceptual son:

- Conceptos: palabras o signos con los que se expresan regularidades correspondientes a imágenes mentales. Hacen referencia a acontecimientos u objetos. Están ubicados dentro de una figura geométrica y se ponen como nodos en los mapas conceptuales.

- Palabras de enlace: sirven para unir los conceptos e indicar el tipo de relación que se establece entre ellos, ya que generan proposiciones parecidas pero no idénticas. Las palabras se anotan en las líneas que unen a dos nodos

- Proposiciones: son dos o más términos conceptuales unidos por palabras para formar una unidad semántica. Deben representar relaciones significativas entre conceptos. En su forma más simple, un mapa conceptual constaría tan sólo de dos conceptos unidos por una palabra de enlace para formar una proposición

Los mapas de conceptos presentan tres particularidades que los diferencian de otros recursos gráficos usados para representar estructuras de información, y corresponden a:

- Jerarquización: la organización jerárquica que presentan modela el significado de las ideas de manera que encajen en una estructura en la que los conceptos están dispuestos por orden de importancia o de "inclusividad" y así se facilita recordar las ideas esenciales. Este tipo de ordenación sugiere la diferenciación de los conceptos porque muestra interrelaciones conceptuales específicas. La jerarquización permite también subordinar un mapa conceptual general a otro más inclusivo.

- Selección: Son una síntesis o resumen que contiene lo más significativo de un tema. Se pueden elaborar sub-mapas que vayan ampliando diferentes partes o subtemas del tema principal.

- Visualización: Un mapa conceptual es conciso y muestra las relaciones entre las ideas principales de un modo simple y rápidamente asimilable, aprovechando la capacidad humana para la representación visual.

En consecuencia, los mapas conceptuales proporcionan el modelo teórico necesario, así como los métodos y técnicas pertinentes para modelar y construir colecciones de objetos de información atendiendo a criterios de organización, relación y visualización, en un entorno integrado, y con una interfaz común. La 


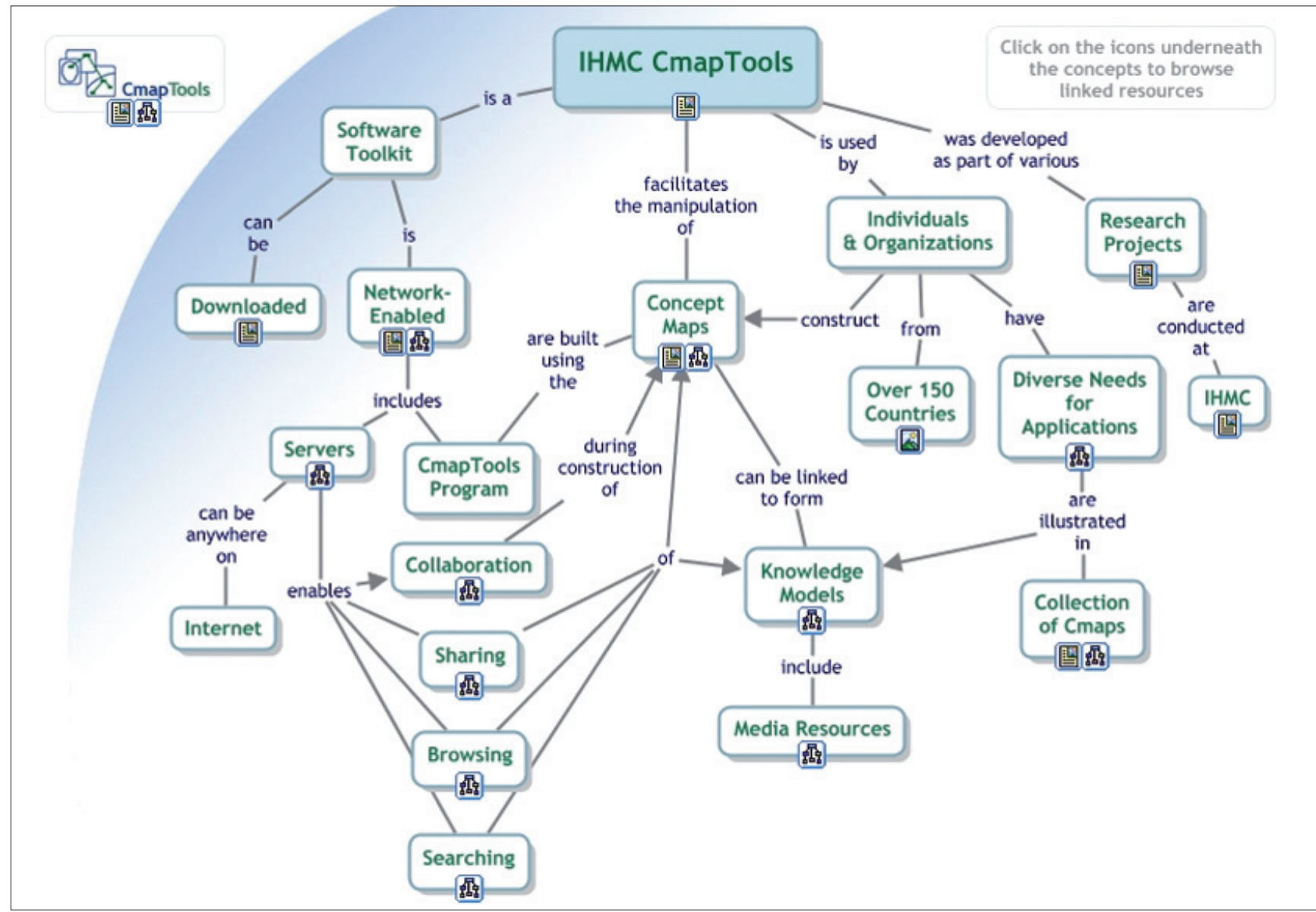

Figura 2. Mapa elaborado con Cmap Tools http://cmap.ihmc.us

revisión de los trabajos presentados en las International Conference on Concept Mapping permite afirmar que existe un creciente interés por usar los mapas de conceptos en entornos de gestión y recuperación de información, con un aumento progresivo de las investigaciones en este campo. Cabe entonces plantear si las herramientas de software disponibles implementan por completo las propiedades del modelo teórico expuesto, y si son capaces de dar soporte adecuado a las demandas que establece la PIM.

http://cmc.ihmc.us/

\section{Herramientas de software para mapas conceptuales}

Los mapas conceptuales permiten llevar a cabo estrategias activas para crear modelos visuales de dominios de conocimiento mediante estructuras que permiten tanto la organización de ese conocimiento, en sentido genérico, como la navegación entre los elementos componentes del dominio. Esto es posible mediante la representación visual de las relaciones lógicas y semánticas existentes entre esos componentes, que han sido establecidas en virtud de los esquemas de organización, clasificación y jerarquización considerados pertinentes por los creadores y usuarios del mapa conceptual. El software para mapas conceptuales debe dar soporte a las funciones básicas necesarias para crear, gestionar y explotar los mapas y la información y el conocimiento contenidos en los mismos, mediante una interfaz visual interactiva. Los productos son mapas conceptuales que representan dominios de conocimiento, así como otros recursos de información relacionados con el dominio representado.

\section{"En el mercado se puede encontrar un elevado número de aplicaciones bajo la denominación de mapas mentales o mapas conceptuales"}

En el mercado se puede encontrar un elevado número de aplicaciones bajo la denominación de mapas mentales o mapas conceptuales. En realidad, y como se ha indicado en el apartado anterior, no se trata del mismo tipo de mapas, ya que los primeros suponen una simplificación de las propiedades semánticas y de tratamiento de información que conllevan los mapas conceptuales. Por esta razón, es mucho menor el número 
de aplicaciones que responden a la etiqueta de mapas conceptuales. La herramienta de referencia más avanzada y extendida para la creación y gestión de mapas conceptuales es actualmente IHMC Cmap Tools, elaborada por el Institute for Human and Machine Cognition, de Florida. La aplicación permite generar mapas conceptuales que respetan el modelo propuesto por Novak (Novak; Cañas, 2008; Leake et al., 2003).

Para el propósito de este trabajo, se han tenido en cuenta aplicaciones que han sido reconocidas como capaces de elaborar y gestionar mapas conceptuales, y que se han publicado bajo licencias de software libre. Se ha seleccionado este tipo de licencias dada la creciente extensión de las mismas, el interés que despiertan, la disponibilidad de prestaciones, y la posibilidad de acceso y uso libre y gratuito por cualquier usuario o servicio de información. Cmap Tools se ha tomado como referencia para la selección y comparación de las aplicaciones valoradas, ya que proporciona soporte al modelo teórico de mapas conceptuales. En el análisis se han incluido aquellas que ofrecían, al menos teóricamente, prestaciones similares a las de Cmap Tools. Se han excluido aquellas capaces de generar representaciones visuales de mapas conceptuales, pero que carecen de las prestaciones necesarias para su edición interactiva. Las estudiadas (véase tabla 1) se detallan en el siguiente párrafo.

VUE (Visual Undertanding Environment) es un software de la Tufts University, que extiende el modelo de mapas conceptuales con capacidades de web semántica, y cuyo principal objetivo es la preparación de colecciones de objetos digitales, orientados a la docencia y a la investigación. FreeMind es un clásico de los mapas mentales, con una amplia base de usuarios y desarrolladores, que amplia sus prestaciones merced a una arquitectura de plugins y complementos. Las valoraciones de usuarios y especialistas lo sitúan al nivel de MindManager, la más reconocida de las aplicaciones privativas. Xmind es un producto de la empresa Xmind, que ha decidido recientemente liberarlo como software libre. Tras ello, se ha producido un notable auge de usuarios y colaboradores en su elaboración, ya que se trata de una herramienta madura y de calidad. MindRaider es, en realidad, un anotador semántico con la capacidad de generar y anotar representaciones visuales, que incluye la posibilidad de generar mapas conceptuales desde contenidos semánticos etiquetados.

\section{Hacia una evaluación cualitativa}

Un vez establecido en apartados anteriores que el modelo teórico de los mapas conceptuales responde a las características que muestran los procesos genéricos de representación y gestión de información personal, y que existen herramientas de software que permiten la implementación de espacios digitales de información basados en mapas conceptuales, se ha llevado a cabo un proceso de análisis dentro de un marco cualitativo de prestaciones. La finalidad de este análisis es establecer si estas aplicaciones, en su formulación actual, responden a todas y cada una de las prestaciones necesarias para gestionar eficaz y eficientemente la información personal. La evaluación se ha llevado a cabo en tres fases:

a. Diseño de una colección de información personal genérica, que incluye:

- Documentos ofimáticos en varios formatos

- Mensajes de correo electrónico

- Ficheros gráficos de contenido estático y diná$\operatorname{mico}$

- Marcadores a recursos de información externa

b. Construcción de la colección en las diferentes aplicaciones seleccionadas, añadiendo:

- Identificación de relaciones

- Etiquetado significativo de conceptos y objetos o elementos de información

- Etiquetado significativo de relaciones

- Anotaciones sobre conceptos, relaciones y elementos

- Etiquetado o anotaciones sobre recursos enlazados

c. Revisión de prestaciones de soporte a la gestión de la colección, atendiendo a:

- Creación de mapas de segundo nivel o combinación/enlace con otros mapas

\begin{tabular}{|l|l|l|l|}
\hline \multicolumn{1}{|c|}{ Herramienta } & \multicolumn{1}{|c|}{ Plataforma } & Versión & \multicolumn{1}{c|}{ url } \\
\hline Freemind & GNU/Linux, Mac OS X, Windows & 0.8 .1 & http://freemind.sourceforge.net/ \\
\hline Xmind & GNU/Linux, Mac OS X, Windows & 3.0 .3 & http://www.xmind.net/ \\
\hline MindRaider & GNU/Linux, Java, Windows & 7.6 & http://mindraider.sourceforge.net/ \\
\hline $\begin{array}{l}\text { Visual Undesrtanding } \\
\text { Environment, VUE }\end{array}$ & GNU/Linux, Mac OS X, Java, Windows & 2.3 & http://vue.tufts.edu/ \\
\hline
\end{tabular}

Tabla 1. Herramientas de software para mapas conceptuales 
- Visualización de zoom progresivo

- Disponibilidad de motor de búsqueda interno

- Formatos de intercambio de información

- Uso de etiquetados semánticos

El resumen de resultados se ha recogido en la tabla 2. La primera fase se ha llevado a cabo sobre una colección en la que se han combinado los tipos de documentos más comunes en los escritorios de la mayoría de los usuarios. Ninguna de las cinco herramientas analizadas proporciona soporte nativo para acceder directamente al contenido de los documentos, ya que sólo actúan como intermediarios. Sin embargo, en un escritorio correctamente configurado las herramientas son capaces de encontrar y lanzar la aplicación correspondiente para cada tipo de objeto de información. La colección se gestiona sin problemas técnicos en plataformas GNU/Linux, Mac OS X y Windows. En este caso, el problema ha consistido en los formatos de los buzones de correo, ya que algunas aplicaciones propietarias no utilizan estándares para los mismos. Tampoco ha sido posible acceder a la información a nivel de mensaje individual, lo que es evidentemente un serio inconveniente para aquellos usuarios que no usen estra- tegias alternativas, o cuyo trabajo se organice principalmente alrededor del correo electrónico. Esta cuestión plantea el nivel de relación que permiten establecer las aplicaciones, ya que cada concepto puede enlazarse a un documento o recurso, pero no a partes componentes del mismo. Las herramientas llevan a identificar y enlazar directamente concepto con recurso y en algunas de ellas, como Xmind, con un único recurso. Esta limitación funcional, que no teórica, restringe las posibilidades de enlazar los conceptos con todos los posibles documentos de referencia, o con las secciones específicas de los mismos. La conexión entre el mapa conceptual y el espacio de información digital no responde entonces ni al modelo teórico ni a las necesidades reales de los usuarios.

La segunda fase ha consistido en la elaboración de un modelo de información personal como mapa conceptual, utilizando las prestaciones de cada una de las herramientas. Todas las aplicaciones usadas han permitido crear el mismo mapa, estableciendo las relaciones entre los conceptos. Sin embargo, Freemind no ha permitido etiquetar las relaciones, lo que no hace posible reflejar la semántica de las relaciones entre conceptos y objetos de información. El etiquetado es complicado

\begin{tabular}{|c|c|c|c|c|}
\hline & Freemind & Xmind & MindRaider & VUE \\
\hline \multicolumn{5}{|l|}{ Diseño de colección de información personal } \\
\hline Formatos ofimáticos & SI & SI & SI & SI \\
\hline Correo electrónico & NO & NO & NO & NO \\
\hline Formatos gráficos & SI & SI & SI & SI \\
\hline Recursos externos & SI & SI & SI & SI \\
\hline Enlazado múltiple & NO & NO & SI & SI \\
\hline \multicolumn{5}{|l|}{ Construcción de la colección } \\
\hline Identificación de relaciones & NO & NO & NO & NO \\
\hline Etiquetado de conceptos & SI & SI & SI & SI \\
\hline Etiquetado de relaciones & NO & SI & NO & SI \\
\hline Anotaciones sobre conceptos & SI & SI & SI & SI \\
\hline Anotaciones sobre relaciones & NO & NO & NO & NO \\
\hline Etiquetado o anotaciones sobre recursos enlazados & NO & NO & NO & SI \\
\hline \multicolumn{5}{|l|}{ Gestión de la colección } \\
\hline Enlace de mapas por niveles & SI & SI & SI & SI \\
\hline Visualización por zoom & NO & NO & SI & SI \\
\hline Motor interno de búsqueda & SI & SI & SI & SI \\
\hline Formatos de intercambio de información & SI & SI & SI & SI \\
\hline Etiquetado $\mathrm{xml}$ & NO & NO & SI & SI \\
\hline Etiquetado rdf & NO & NO & SI & SI \\
\hline Ontologías & NO & NO & NO & SI \\
\hline
\end{tabular}

Tabla 2. Resumen de resultados. 
en MindRaider, a pesar de que utiliza rdf como capa semántica. Se ha querido distinguir aquellas herramientas que permiten, además del etiquetado significativo de nodos, relaciones y objetos de información, la posibilidad de utilizar etiquetado descriptivo libre, al estilo de las folksonomías de usuario final. Sólo VUE, MindRaider y XMind ofrecen esta prestación. Si se acepta que el etiquetado descriptivo es una necesidad actual de los usuarios, las prestaciones técnicas comienzan a limitar la utilidad de varias aplicaciones. La posibilidad de realizar anotaciones de usuario para los componentes del mapa de conceptos es una prestación disponible en todas las herramientas.

La tercera fase ha incidido en prestaciones que, si bien no son imprescindibles para la creación de mapas de conceptos, si son necesarias cuando éstos se usan para representar colecciones de información personal. Todas las herramientas hacen posible enlazar mapas mediante anidamiento, lo que facilitaría organizar la información según un modelo de capas o niveles. Sin embargo, no todas ellas lo complementan con una visión de zoom dinámico, sólo disponible en MindRaider y VUE. Si las organizaciones de información son complejas, esta limitación en los mecanismos de visualización dificulta en primer lugar la navegación por grandes espacios de información y, en segundo, la comprensión del esquema general de organización. En lo que respecta a mecanismos de búsqueda de información textual, todas las herramientas buscan en el texto de conceptos, etiquetas y anotaciones, pero falta en todas ellas integración con

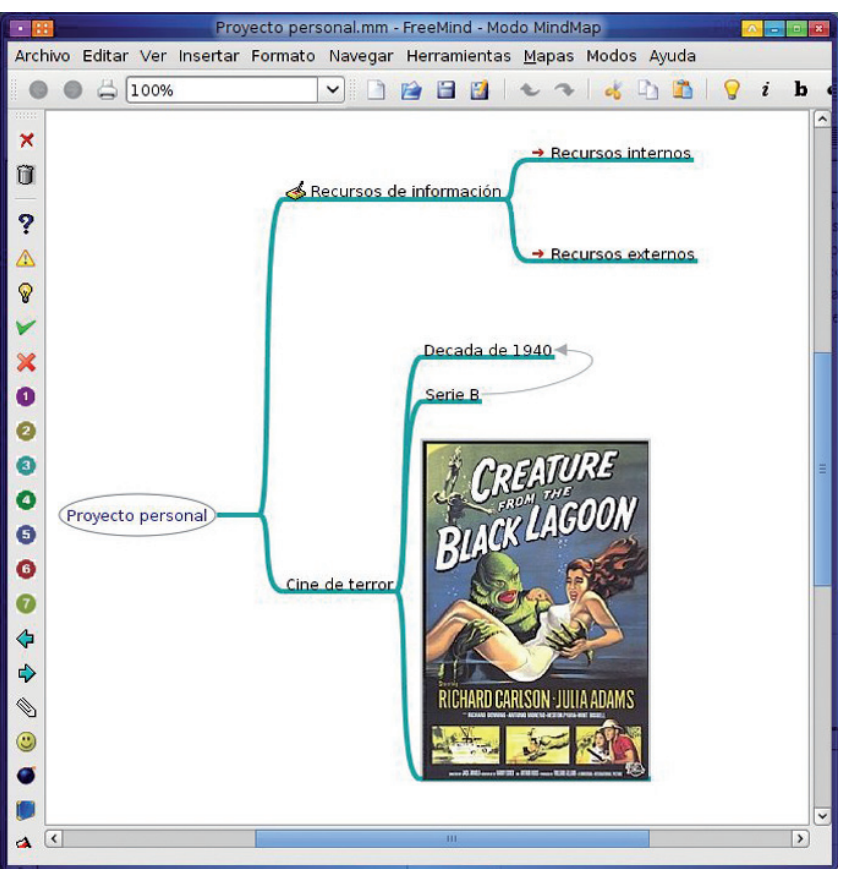

Figura 3. Edición de sección de mapa en Freemind

aplicaciones de búsqueda de escritorio, que permitan acceder al contenido de los documentos o marcadores externos referenciados en la colección. Sólo VUE presenta la posibilidad de extender las búsquedas a otros motores y recursos disponibles en internet.

Mención aparte merece la implementación de los formatos para el intercambio de información. Todas las aplicaciones tienen opciones de importación y exportación de mapas, pero o están orientadas a mapas mentales

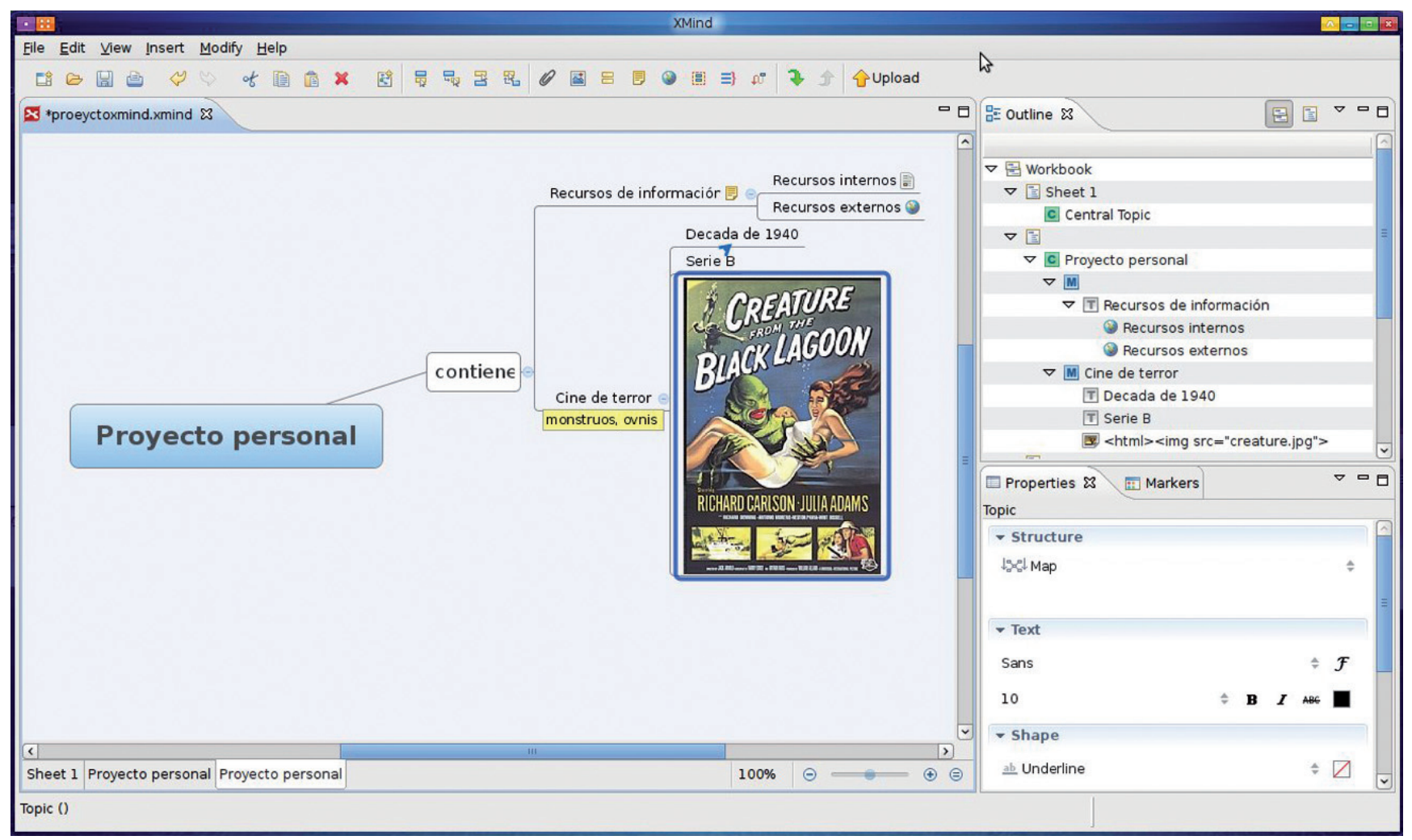

Figura 4. Edición de sección de mapa en Xmind 


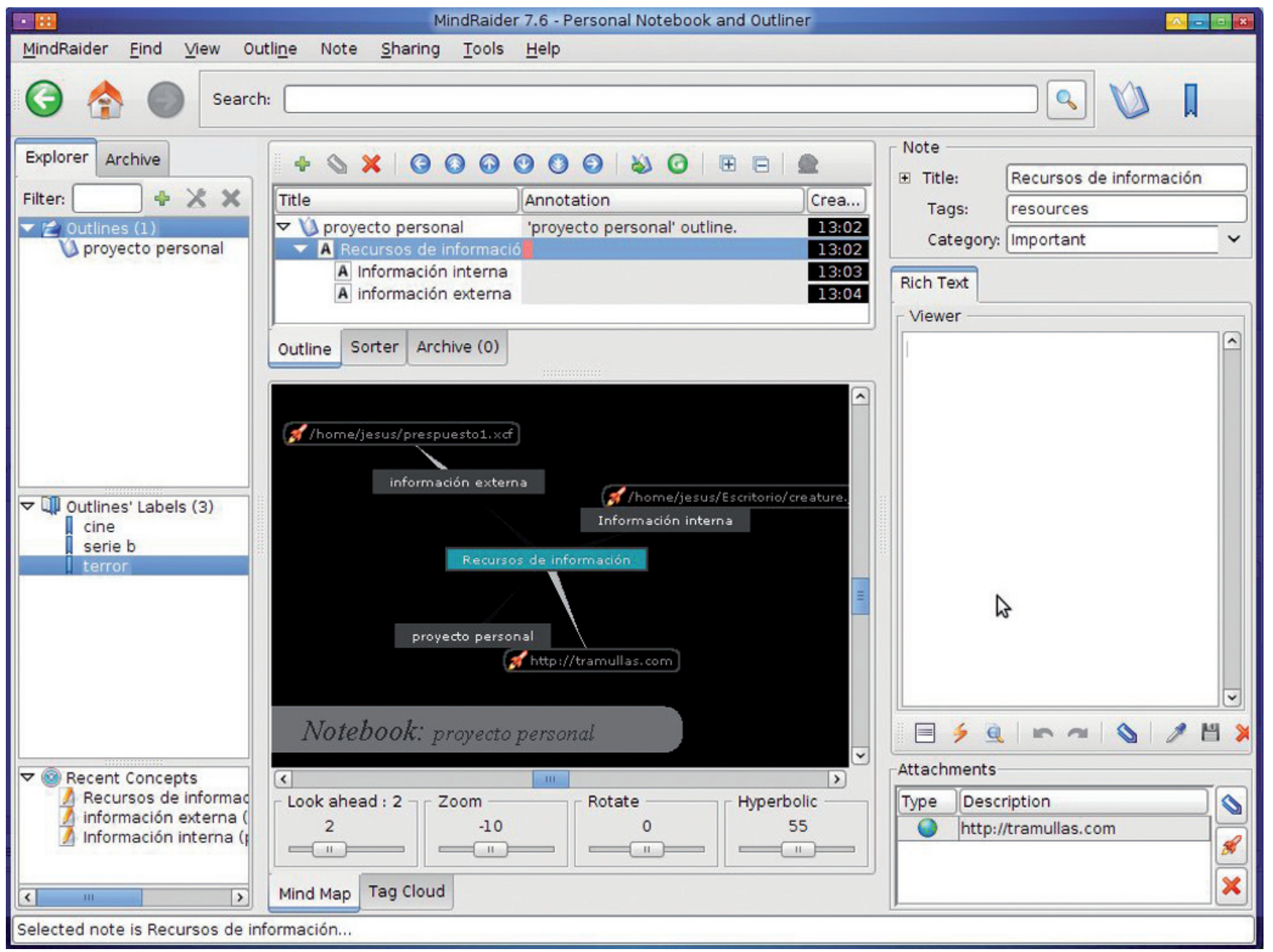

Figura 5. Edición de sección de mapa en MindRaider

(hacia el estándar de facto de MindManager), como es el caso de Freemind y XMind, o los etiquetados que podemos considerar semánticos son limitados, como MindRaider, que sólo ofrece opml y un limitado rdf. Esto enlaza con las funcionalidades para integrar etiquetados semánticos. Exceptuando VUE, que contempla el uso de ontologías, o un plugin complementario para gestionar Topic Maps que se puede añadir a FreeMind, o la posibilidad de usar hojas de estilo para reaprovechar etiquetados en MindRaider, las prestaciones que se podrían calificar como semánticas no están disponibles en estas herramientas. No puede aceptarse que un mero etiquetado en xml o sus derivados convierte a una aplicación en semántica: "xml will continue to play an important role in the development of the semantic web. However, it does not provide a full solution to the requirements of the semantic web [...] resources on the web need to be represented in or annotated with structured machineunderstandable descriptions of their contents and relationships, using vocabularies and constructs that have been explicitly and formally defined with a domain ontology” (Lu; Dong; Fotouhi, 2002).

Tras la revisión efectuada se puede afirmar que las herramientas de software para mapas conceptuales no están preparadas, en sus versiones actuales, para gestionar información personal de manera eficiente. Aunque en ocasiones se han presentado como muy adecuadas para organizar visualmente gran cantidad de información, lo cierto es que las carencias que muestran en mecanismos de visualización, de etiquetado semántico y de integración con otras herramientas de escritorio limitan severamente su potencial. Sólo VUE ofrece las funcionalidades establecidas como básicas para crear espacios de información personal.

\section{Conclusiones}

En 1987, Dow proponía que los bibliotecarios deberían extender su actividad hacia los usuarios para actuar como consejeros profesionales en la PIM. Desde la perspectiva del tiempo transcurrido, hay que destacar que la PIM se ha extendido, consciente o inconscientemente, a todos los usuarios de los servicios de la sociedad de la información. Una de las grandes beneficiadas es la alfabetización informacional, ya que la PIM puede aportar a los usuarios técnicas y estrategias para identificar y usar eficientemente la información digital en cualquier contexto, tanto público como privado. 
Sin embargo, no puede hablarse de un estado "normal" o "consolidado" de la PIM. Al tratarse de una actividad individual, creativa y adaptable, no hay metodologías comunes, más allá de pautas, experiencias y recomendaciones, que deberían adaptarse para cada caso. Los estudios de usuario demuestran la gran cantidad de comportamientos diferentes, dependientes de numerosos factores. A su vez la diversidad de objetos de información a los que atender provocan la denominada fragmentación de la información, las herramientas disponibles empiezan a ser complicadas en su comprensión y funcionamiento, y no ofrecen soluciones globales que permitan la deseable integración. Las capacidades de gestionar información digital de manera integrada son todavía bastante limitadas.

En el caso de las herramientas de software para mapas conceptuales, si bien el modelo teórico subyacente se ajusta adecuadamente a la organización y acceso a una colección de objetos de información digital, las prestaciones técnicas reales no son suficientes para los requerimientos actuales de gestión de información.

\section{Bibliografía}

Barreau, Deborah et al. "Introduction to keeping, refinding and sharing personal information". ACM Transactions on Information Systems, 2008, v. 26, n. 4.

Barreau, Deborah. “"Gestión” de información personal, no sólo "recuperación" de información personal”. El profesional de la información, 2009, julio-agosto, v. 18, n. 4, pp. 361-364.

Bergman, Ofer; Beyth-Marom, Ruth; Nachmias, Rafi. "The user-subjective approach to personal information management systems". Journal of the American Society for Information Science and Technology, 2003, v. 54, n. 9 , pp. 872-878.

Bergman, Ofer; Beyth-Marom, Ruth; Nachmias, Rafi. “The user-subjective approach to personal information management systems design: evidence and implementations". Journal of the American Society for Information Science and Technology, 2008, v. 59, n. 2, pp. 235-246.

Bergman, Ofer et al. "Improved search engines and navigation preference in personal information management". ACM Transactions on Information Systems, 2008, v. 26, n. 4.

Bernstein, Michael; Van Kllek, Max; Karger, David; Schraefel, Monica M. C. "Information scraps: how and why information eludes our personal information management tools". ACM Transactions on Information Syste$m s, 2008$, v. 26 , n. 4.

http://people.csail.mit.edu/msbernst/papers/TOIS-final-with-refs.pdf

Boardman, Richard. Improving Tool Support for Personal Information Management. Doctoral Thesis, London: Imperial College, Univ. of London, 2004.

Boardman, Richard; Sasse, M. Angela. "Stuff goes into the computer and doesn't come out. A croos-tool study of personal information management". CHI, 2004, pp. 583-590.

http://www.iis.ee.ic.ac.uk/ rick/research/pubs/boardman-chi04.pdf

Bruce, Harry. "Personal, anticipated information need". Information research, 2005, v. 10, n. 3.

http://informationr.net/ir/10-3/paper232.html

Bruce, Harry; Jones, William; Dumais, Susan. "Information behaviour that keeps found things found". Information research, 2004, v. 10, n. 1. http://informationr.net/ir/10-1/paper207.html

Buzan, Tony. The mind map book. London: Penguin, 2000.
Colomb, Robert M. Information spaces: the architecture of cyberspace. London: Springer, 2002.

Cutrell, Edward; Dumais, Susan T.; Teevan, Jaime. "Searching to eliminate personal information management". Communications of the ACM, 2006, v. 49, n. 1, pp.58-64.

Dow, Elizabeth H. "Personal information systems: the library role". Library journal, 1987, v. 112, n. 18, pp. 29-32.

Elsweiler, David; Ruthven, Ian; Jones, Christopher. "Towards memory supporting personal information management tools". Journal of the American Society for Information Science and Technology, 2007, v. 58, n. 7, pp. 924-946.

Gwizdka, Jacek; Chignell, Mark. "Individual differences". En: Jones, William; Teevan, Jaime (eds.). Personal information management. Washington: Washington University Press, 2007.

Jones, William. "Finders, keepers? The present and future perfect in support of personal information management”. First Monday, 2004, v. 9, n. 3. http://firstmonday.org/htbin/cgiwrap/bin/ojs/index.php/fm/article/ view/1123/1043

Jones, William. Keeping found things found: the study and practice of personal information management. Morgan Kauffman, 2007a.

Jones, William. "Personal information management". En: Cronin, Blaise (ed.), Annual Review of Information Science and Technology (ARIST), 2007b, v. 41.

Jones, William; Teevan, Jaime. (eds.). Personal information management. Washington: Washington University Press, 2007.

Karger, David R.; Jones, William. "Data unification in personal information management". Communications of the ACM, 2006, v. 49, n. 1, pp.77-82.

Khoo, Christopher S. G.; Luyt, Brendan; Ee, Caroline; Osman, Jamila; Lim, Hui-Hui; Yong, Sally. "How users organize electronic files on their workstations in the office environment: a preliminary study of personal information organization behaviour". Information research, 2007, v. 11, n. 2. http://informationr.net/ir/12-2/paper293.html

Leake, David B. et al. "Aiding knowledge capture by searching for extensions of knowledge models". En: Proceedings of the 2nd International Conference on Knowledge Capture. ACM, 2003, pp. 44-53.

Lu, Shiyong; Dong, Ming; Fotouhi, Farshad. "The semantic web: opportunities and challenges for next-generation web applications". Information research, 2002, v. 7, n. 4.

http://informationr.net/ir/7-4/paper134.html

McKnight, Cliff. "The personal construction of information space". Journal of the American Society for Information Science, 2000, v. 51, n. 8, pp. 730-733.

Novak, Joseph D.; Cañas, Alberto J. The theory underlying concept maps and how to construct and use them. Pensacola: Florida Intitute for Human and Machine Cognition. Technical report IHMC Cmap Tools 2006-01 rev 2008-01.

http://cmap.ihmc.us/Publications/ResearchPapers/TheoryCmaps/ TheoryUnderlying ConceptMaps.htm

Sauermann, Leo. "The semantic desktop - a basis for personal knowledge management". En: Proceedings of I-KNOW'05 5th International Conference on Knowledge Management, 2005, pp. 294-301.

http://www.dfki.uni-kl.de/ sauermann/papers/Sauermann2005b.pdf

Sauermann, Leo; Van-Elst, Ludger; Dengel, Andreas. "PIMO - a framework for representing personal information models". En: I-Semantics $073 \mathrm{rd}$ International Conference on Semantic Technologies, Graz, 2007. http://www.dfki.uni-kl.de/ sauermann/papers/sauermann+2007b.pdf

Jesús Tramullas, Piedad Garrido-Picazo, Ana-I. Sánchez-Casabón, Universidad de Zaragoza.

tramullas@unizar.es

piedad@unizar.es

asanchez@unizar.es 